\title{
COMPARISON OF WRAP AROUND SCREENS AND HMDS ON A DRIVER'S RESPONSE TO AN UNEXPECTED PEDESTRIAN CROSSING USING SIMULATOR VEHICLE PARAMETERS
}

\author{
Danielle Filio, Lynn Dony, Diego Gonzalez and Michele Oliver \\ School of Engineering, University of Guelph, Guelph, Ontario, Canada \\ Email: tfilio@uoguelph.ca
}

\begin{abstract}
Summary: Driving simulators are typically used when analyzing hazardous and collision events, as dangerous drives can be performed in controlled environments without compromising driver safety. Most driving simulators use a form of wrap around screens to project the simulation as they provide a wide field of view for the user creating a more realistic experience. However, this visual modality is costly and not practical for smaller workspaces. Recent advancements in head mounted display (HMD) technology may make them a better alternative to wrap around screens, but studies have yet to compare the two visual modality effects on driver performance in hazardous scenarios. In this study, drivers completed two drive simulations, one using wrap around screens and the other using a commercially available HMD. Each simulation contained two different unexpected pedestrian crossings in which the perception-response time and brake movement time of the driver was assessed. Average vehicle speed and standard deviation of lateral position were also examined. Perception-response time was significantly longer for drivers when wearing the HMD than when using wrap around screens. There were also significant differences in vehicle speed during driver perception-response time and brake movement time between display modalities but standard deviation of lateral position only had significant differences during perception-response times. Further advancements in HMD technology are needed before they can provide an adequate alternative to wrap around screens when analyzing driver response scenarios.
\end{abstract}

\section{OBJECTIVES}

There is a need to analyze hazardous and collision scenarios for road safety, driver training programs and vehicle and roadway design (Chan, Pradhan, Pollatsek, Knodler, \& Fisher, 2010; Mueller, Stanley, Martin, \& Gallagher, 2014; Rosey \& Auberlet, 2014). Studying these situations in high-speed, in-vehicle experiments may provide insight to driver performance, however, these experiments can pose great risk to the safety of the participant as well as the safety of those in the surrounding test environment (Rosey \& Auberlet, 2014). This makes the use of driving simulation desirable as the experiment can be controlled for repeatability while maintaining participant safety (Rosey \& Auberlet, 2014). One concern with using driving simulations to analyze hazard response is whether both the performance and behavioural responses of the driver can be adequately measured (Chan et al., 2010). Driving simulators are known to be adequate in analyzing driver performance (Wang, Peng, Liang, Zhang, \& Wu, 2007), but a study conducted by Chan et al. (2010), found that driving simulators can also be adequate in analyzing driver behaviour during hazard response scenarios. The authors found this to be useful to driver training programs as well as to vehicle and road designers (Chan et al., 2010). 
One of the main elements of simulator design is the visual cueing system, which allows the driver to see their progress through the simulation (Fisher, Rizzo, Caird, \& Lee, 2011a). Visual cueing systems have progressed from using analog film projection in the earlier years to the use of 3D graphics cards and large screens (Fisher et al., 2011a). One of the biggest issues of the visual system is the resolution of the projection modality, as technologies that allow for higher resolution displays are more costly (Fisher et al., 2011a). Most driving simulators project the simulation using computers or wrap around screens, which can range in size, resolution and refresh rate (Chan et al., 2010; Rosey \& Auberlet, 2014). Some disadvantages to wrap around screens include their high cost and large space requirements (Fisher, Rizzo, Caird, \& Lee, 2011b).

Recent advancements in head mounted display (HMD) technology, including a reduction in both size and cost has made them an enticing alternative to wrap around screens (Fisher et al., 2011b; Patterson, Winterbottom, \& Pierce, 2006; Simone, Schultheis, Rebimbas, \& Millis, 2006). One of the current issues with HMD technology is that most devices offer a smaller field of view than that capable with wrap around screens (Fisher et al., 2011b). While studies have linked reduced field of views to decreases in simulator sickness, the reduction in field of view may have an impact on driver hazard response (Fisher, Rizzo, Caird, \& Lee, 2011c, 2011d). Meaningful response differences can be in the millisecond range suggesting that the even minimal differences in driver performance parameters induced by the reduced field of view could make the use of HMD technology undesirable (SAE International, 2015). The average horizontal field of view of a person is $200^{\circ}$, with $120^{\circ}$ of that range seen by both eyes (Fisher et al., 2011b; Patterson et al., 2006).

When analyzing driver response to an event, most studies report reaction time and trajectory control, specifically perception-response time, vehicle speed and standard deviation of lateral position of the vehicle (Fisher et al., 2011c, 2011d; Mueller et al., 2014; Rosey \& Auberlet, 2014). Perception response time encompasses the perception, recognition and initial action of the driver when reacting to a hazard (SAE International, 2015).

The objective of this study was to compare the effects of wrap around screens to a commercially available HMD on driver response in a driving simulator during unexpected pedestrian crossings. The variables analyzed were perception-response time, brake movement time, standard deviation of lateral position of the vehicle and vehicle speed. It was expected that the perception-response time would be longer when the driver used the HMD due to the reduced field of view. If significant differences between driver responses existed, then further advancements in HMD technology, such as an increased field of view, would be required before HMDs could be confidently used instead of wrap around screens during driving simulator experiments.

\section{METHODOLOGY}

\section{Participants}

After providing informed consent, 29 drivers (22 male, 7 female) between the ages of 18 and 31 $($ Mean $=23, \mathrm{SD}=3)$ participated in this study. Exclusion criteria included those who 
experienced symptoms of simulator adaptation syndrome (Kennedy, 1993), and those who did not have a minimum of a class G2 Ontario driver's license or equivalent.

\section{Equipment}

The driving simulator used in this study was a fixed-based, OKTAL full-scale Pontiac G6 convertible (OKTAL, Toulouse, France). The simulation was projected onto six rectangular 7'x7'2'" screens which were wrapped around the vehicle, providing a $300^{\circ}$ field of view. The image projected on the screens had a resolution of 1920 by 2160 pixels and refresh rate of $60 \mathrm{~Hz}$. The other visual modality used in this study was an Oculus Rift" ${ }^{\mathrm{TM}}$ DK2 (Oculus VR, CA) HMD. This HMD had a resolution of $960 \times 1080$ pixels, a refresh rate of $60 \mathrm{~Hz}$ and provided the participant with a 100 degree field of view. The screen is divided into two identical images of the virtual environment, providing the driver with stereovision. The Oculus SDK was used to access the position and orientation of the device in real time to update the view presented to the driver according to the direction and motion of their head.

\section{Simulator Scenarios}

The road network used for the driving simulations was generated using the elevation of roadways in the Guelph, Ontario, Canada area. The road network was comprised of both a city and a rural landscape. A path was established between the city and the country with road signs along the roadway directing the participant along the appropriate path. If the participant did not see the signs or was unsure of which path to take, the experimenter directed them. The landscape where the simulation would start was randomized. If the starting point of the simulation was in the city area, then the simulation ended in the country area and vice versa.

Each simulation contained two different unexpected pedestrian crossings, one in the city and one in the country. For both environments, this event occurred on a segment of straight roadway where the pedestrian initially faced the direction parallel to the vehicle's lane. The pedestrian would then make a 90 degree turn toward the roadway and walk in straight line, in front of the vehicle's path until reaching the sidewalk on the other side.

\section{Experimental Procedures}

Prior to the experiment trials, each participant performed two familiarization drives, one using the wrap around screens and the other using the HMD. Each experiment consisted of two trials, one where the simulation was projected on the wrap around screens and the other on the HMD. The order of visual modality used was randomized for each participant. The duration of the drive in each trial was approximately 10 minutes. Vehicle data were recorded during the trials using OKTAL SCANeRStudio ${ }^{\mathrm{TM}}$ (OKTAL, Toulouse, France) at a $100 \mathrm{~Hz}$ sampling rate.

\section{Data Processing}

The data from the simulations were processed using custom MATLAB ${ }^{\text {TM }}$ code (The MathWorks Inc, MA, USA). The variables calculated were the driver perception-response time, driver brake time, vehicle speed and standard deviation of lateral position. 
Perception-Response Time. The start of the perception-response time was defined as the point at which the pedestrian began their $90^{\circ}$ turn to cross the road. This was the first opportunity that the driver had to perceive the hazard. The end point was defined as the point at which the driver initially applied force to the brake pedal, as indicated by the first non-zero brake force recorded at the pedal.

Brake Movement Time. The brake movement time was defined as the end of the driver perception-response time to the time when the driver reached the maximum force applied to the brake pedal during the pedestrian crossing.

Vehicle Speed. The vehicle speed was obtained from the Oktal SCANeRStudio ${ }^{\mathrm{TM}}{ }_{\text {software. The }}$ mean of the vehicle speed during both the driver perception response time and brake movement time was calculated. In addition, the mean vehicle speed during a 10 second segment was calculated where the participant was driving on a straight portion of road. This was done in order to compare the vehicle speed during the driver perception response time and brake movement time.

Standard Deviation of Lateral Position (SDLP). Lateral position was obtained from the Oktal SCANeRStudio ${ }^{\mathrm{TM}}$ software and exported to Matlab ${ }^{\mathrm{TM}}$ to calculate the standard deviation. The SDLP was calculated for driver perception response time, brake movement time and the 10 second straight segment. The equation for standard deviation used in the custom Matlab ${ }^{\mathrm{TM}}$ code is given below.

$$
S D L P=\sqrt{\frac{1}{N-1} \sum_{i=1}^{N}\left(x_{i}-x_{\text {mean }}\right)^{2}}
$$

Where $\mathrm{x}_{\mathrm{i}}$ is lateral position and $\mathrm{N}$ is the number of lateral position samples over the time period.

\section{Statistical Analyses}

Analysis of variance procedures were performed $(\mathrm{p} \leq 0.05)$ on the dependent variables which included the Perception Response Time and Brake Movement Time. The independent variable was Display Modality (HMD or Wrap Around Screens). Further ANOVAs were performed on Vehicle Speed and Standard Deviation of Lateral Position where data were analyzed separately during the driver perception response time, brake movement time and a 10 second segment where the participant was driving on a straight portion of road. Again, the independent variable was Display Modality. All statistical analyses were performed using Minitab ${ }^{\mathrm{TM}}$ version 17.2.1 (Minitab Inc., State College, PA). When assumptions of normality were not met, data were transformed using Johnson Transformations.

\section{RESULTS AND DISCUSSION}

Perception-response time was significantly longer $(\mathrm{F}(1,28)=4.13 ; \mathrm{p}=0.05)$ when drivers were wearing the HMD than using the wrap around screens by approximately 0.5 seconds as shown in Figure 1. No significant differences were found between the two visual modalities in brake movement times. This suggests that the reduced field of view offered by the HMD impacted the time it took for the driver to recognize that the pedestrian was going to cross the roadway. The 
pedestrian, who was initially standing on the sidewalk, could have been outside of the field of view offered by the HMD but not outside of the field of view of the wrap around screens. During the brake movement time, the driver is reacting to the pedestrian by applying force to the brake pedal. This indicates that during this time, the pedestrian is within their field of view, explaining the lack of significant differences between the visual modalities during the brake movement time.

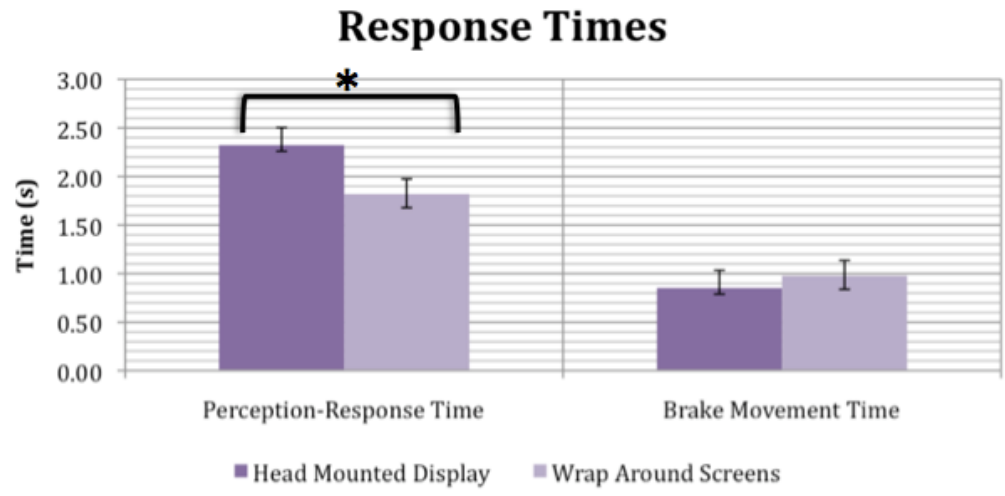

Figure 1. Comparison of average response times (mean \pm standard error of the mean) between the head mounted display and wrap around screens (*Significantly different $\mathbf{p} \leq \mathbf{0 . 0 5}$ )

Vehicle speed was significantly higher during both response times (Perception-Response $\mathrm{F}(1,28)=12.24 ; \mathrm{p}=0.002$; Brake Movement Time $\mathrm{F}(1,28)=8.25 ; \mathrm{p}=0.008$ ) when the driver was using the wrap around screens over using the HMD (Figure 2). The driver may have been more comfortable using the wrap around screens than the HMD, explaining the greater vehicle speeds using the wrap around screens during the response to the hazard. Straight portions of driving showed no significant differences in vehicle speeds between the visual modalities.

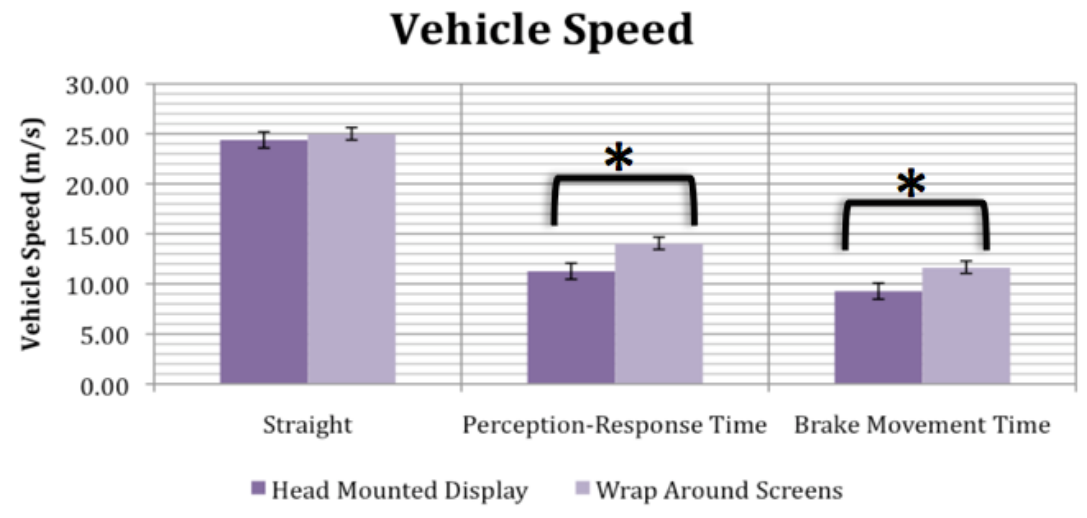

Figure 2. Comparison of vehicle speed (mean \pm standard error of the mean) between the head mounted display and wrap around screens (*Significantly different $p \leq 0.05$ )

SDLP was significantly greater during the driver perception response time $(\mathrm{F}(1,28)=7.48$; $\mathrm{p}=0.01$ ) when using the HMD over the wrap around screens (Figure 3). There were no significant differences in SDLP during the brake movement time period between the two visual modalities. SDLP for straight portions of driving was significantly higher when using the HMD $(F(1,28)=4.10 ; p=0.05)$. This result was somewhat unexpected although when considered in 
concert with the reduced field of view and higher vehicle speeds during the straight portions of driving, the participants may have felt more comfortable driving at faster speeds but may have also experienced a greater workload due to the reduced field of view (Fisher et al., 2011b). This may have made it difficult at higher speeds to maintain a straight course. The differences in SDLP between the modalities during the perception-response time period could be directly related to the differences seen in perception-response time. Following hazard presentation, the longer perception-response time found when wearing the HMD suggests that the driver may have also had a delayed reaction, potentially causing them to compensate by swerving prior to braking. The perception-response time would have to further be broken into perception time and reaction time, to confirm or refute this statement.

\section{Standard Deviation of Lateral Position}

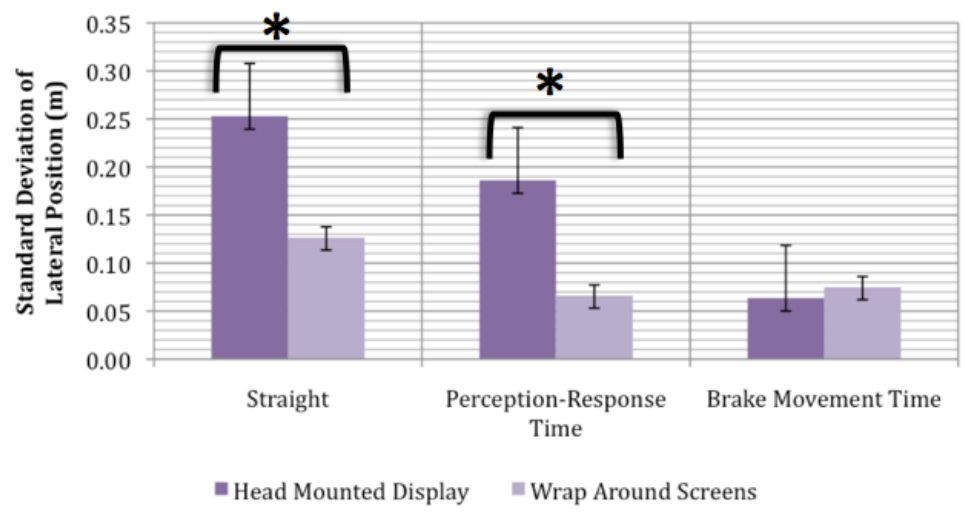

Figure 3. Comparison of standard deviation of lane position (mean \pm standard error of the mean) between the head mounted display and wrap around screens. ( ${ }^{*}$ Significantly different $\left.p \leq 0.05\right)$

\section{CONCLUSION}

The significant differences in perception response time between the two visual modalities suggest that researchers analyzing hazard response should be cautious when selecting a visual modality. Future research should look at comparing HMD technology to wrap around screens that have identical specifications including their refresh rate, field of view and resolution.

Research comparing the two visual modalities is ongoing to determine if there are differences in driver performance at a biomechanical and physiological level. This includes analyzing upper body kinematics and muscle activity through the use of motion capture and surface electromyography technology. This will help to further elucidate the effects of display modality on driver responses to simulated driving conditions.

\section{ACKNOWLEDGEMENTS}

Funding for this research was provided by grants to the second author from the Canadian Foundation for Innovation and Natural Sciences and Engineering Research Council of Canada. 


\section{REFERENCES}

Chan, E., Pradhan, A. K., Pollatsek, A., Knodler, M. A., \& Fisher, D. L. (2010). Are driving simulators effective tools for evaluating novice drivers' hazard anticipation, speed management, and attention maintenance skills? Transportation Research Part F-Traffic Psychology and Behaviour, 13(5), 343-353. https://doi.org/10.1016/j.trf.2010.04.001

Fisher, D. L., Rizzo, M., Caird, J., \& Lee, J. (2011a). A Short History of Driving Simulation. In Driving Simulation For Engineering, Medicine, and Psychology (pp. 2.2 - 2.11). CRC Press: Taylor and Francis Group.

Fisher, D. L., Rizzo, M., Caird, J., \& Lee, J. (2011b). Simulator and Scenario Factors Influencing Simulator Sickness. In Driving Simulation for Engineering, Medicine, and Psychology (pp. 14.13-14.16). CRC Press: Taylor and Francis Group.

Fisher, D. L., Rizzo, M., Caird, J., \& Lee, J. (2011c). Situation Awareness in Driving. In Driving Simulation for Engineering, Psychology, and Medicine (pp. 19.2-19.9). CRC Press: Taylor and Francis Group.

Fisher, D. L., Rizzo, M., Caird, J., \& Lee, J. (2011d). Twelve Practical and Useful Questions about Driving Simulation. In Driving Simulation for Engineering, Medicine, and Psychology (pp. 5.2 - 5.15). CRC Press: Taylor and Francis Group.

Kennedy, R. S., Lane, N. E., Berbaum, K. S., \& Lilienthal, M. G. (1993). Simulator sickness questionnaire: An enhanced method for quantifying simulator sickness. The international journal of aviation psychology, 3(3), 203-220.

Mueller, J., Stanley, L., Martin, T., \& Gallagher, C. (2014). Driving Simulator and Scenario Effects on Driver Response. IIE Annual Conference. Proceedings, 507-513.

Patterson, R., Winterbottom, M. D., \& Pierce, B. J. (2006). Perceptual Issues in the Use of HeadMounted Visual Displays. Human Factors: The Journal of the Human Factors and Ergonomics Society, 48(3), 555-573. https://doi.org/10.1518/001872006778606877

Rosey, F., \& Auberlet, J.-M. (2014). Driving simulator configuration impacts drivers' behavior and control performance: An example with studies of a rural intersection. Transportation Research Part F-Traffic Psychology and Behaviour, 27, 99-111. https://doi.org/10.1016/j.trf.2014.09.009

SAE International. (2015). Operational definitions of driving performance (pp. 1-171). SAE International.

Simone, L. K., Schultheis, M. T., Rebimbas, J., \& Millis, S. R. (2006). Head-mounted displays for clinical virtual reality applications: Pitfalls in understanding user behavior while using technology. Cyberpsychology \& Behavior, 9(5), 591-602.

https://doi.org/10.1089/cpb.2006.9.591

Wang, Y., Peng, P., Liang, L., Zhang, W., \& Wu, S. (2007). Road hazard reaction testing using driving simulation: the novice vs. the experienced drivers. (M. Helander, M. Xie, M. Jaio, \& K. C. Tan, Eds.). New York: IEEE. 